\title{
Regulated cell death in Hetherocephalus glaber
}

\author{
Aleksei Popov \\ ICBFM SB RAS, Novosibirsk, Russia \\ depolice@mail.ru \\ Svetlana Romanenko \\ IMCB SB RAS, Novosibirsk, Russia \\ rosa@mcb.nsc.ru \\ Inna Lavrik \\ Institute of Experimental Internal \\ Medicine, Magdeburg, Germany \\ inna.lavrik@med.ovgu.de
}

\author{
Aleksei Evdokimov \\ ICBFM SB RAS, Novosibirsk, Russia \\ an_evdokimov@mail.ru \\ Vladimir Trifonov \\ IMCB SB RAS, Novosibirsk, Russia \\ vlad@mcb.nsc.ru \\ Elena Ryabchikova \\ ICBFM SB RAS, Novosibirsk, Russia \\ lenryab@niboch.nsc.ru
}

\author{
Irina Petruseva \\ ICBFM SB RAS, Novosibirsk, Russia \\ irapetru@niboch.nsc.ru \\ Olga Koval \\ ICBFM SB RAS, Novosibirsk, Russia \\ o.koval@niboch.nsc.ru \\ Olga Lavrik \\ ICBFM SB RAS, Novosibirsk, Russia \\ lavrik@niboch.nsc.ru
}

\begin{abstract}
Heterocephalus glaber, or the naked mole rat, is the longest-living rodent species that is extraordinary resistant to cancer and aging-related diseases. The molecular basis for the unique phenotypic traits of naked mole-rat has been extensively studied recently, but the role of the programmed cell death in longevity and cancer resistance of naked mole rat is still insufficiently understood. Regulated cell death is a mechanism restricting the proliferation of damaged or premalignant cells which counteracts aging and oncotransformation. In this study, the systematic analysis of naked mole rat fibroblasts cells to undergo DNA-damage-induced apoptosis has been carried out using conventional methods of apoptosis detection. Skin fibroblasts from the naked mole rat were shown to be more resistant to several types of stress effects compared with fibroblasts from the Mus musculus. Naked mole rat cells also exhibit a limited apoptotic response and seem to undergo necrotic cell death under severe stress.
\end{abstract}

Keywords - aging, longevity, naked mole rat, stress resistance, apoptosis

\section{Motivation and Aim}

\section{Motivation}

It was previously demonstrated that vascular endothelial cells in naked mole rat (NMR) are highly resistant to apoptotic stimuli such as $\mathrm{H} 2 \mathrm{O} 2$ treatment or heat exposure compared with mouse cells [1], the ability of NMR cells to undergo apoptosis in response to stress has been studied insufficiently, the details of the processes leading to apoptosis in NMR cells remain unclear. Also, no comprehensive studies of the regulated cell death (RCD) in NMR and its role in cancer development have been conducted yet.

Aim

To determine the features of the regulated cell death in Heterocephalus glaber, we have carried out a systematic comparison of DNA-damage-induced apoptosis in skin fibroblasts from Heterocephalus glaber and Mus musculus.

\section{Methods}

Experiments were provided using NMR skin cell line and 3T3 mouse cell line. Three compounds that are well known to induce three different types of DNA damage agents were used as genotoxic stress agents: methyl methanesulfonate (MMS) [2], 5-fluorouracil (5FU) [3], and etoposide [4]. The resistance of NMR and mouse fibroblasts to stress induced by selected reagents was evaluated using cell metabolic activity assay. FITC Annexin V Apoptosis detection kit and FACSCanto II (BD Biosciences, San Jose, USA) flow cytometer were used to detect cell death. To perform differential study of the development of apoptotic cascade dynamics, caspase activities were measured using the Caspase-Glo 3/7 assay kit according to the manufacturer's instructions (Promega, Madison, WI). Luminescence of the samples was measured using an Infinite M200 plate reader (Tecan, Research Triangle Park, USA). Electron microscopy of ultrathin sections was used to delineate between the types of cell death in NMR and mouse cells. Statistical analysis was performed using Statistica 10 software.

\section{Results}

The comparative studies have revealed that naked mole rat cells are highly resistant to all types of exposures used. The decline in cellular metabolic activity and cell death take place at much higher concentrations of cytotoxic agents. Unlike mouse cells, naked mole rat cells are especially resistant to some proapoptotic reagents (e.g., 5-fluorouracil). The data obtained using various approaches (flow cytometry, electron microscopy, and measuring caspase activity during incubation in the presence of reagents) give grounds for assuming that efficiency of apoptosis activation in the naked mole rat is lower compared to that in mice. When exposed to high-dose toxic agents, the naked mole rat cells preferentially undergo necrotic rather than apoptotic death (unlike mouse cells exposed to the same agents).

\section{ACKNOWLEDGMENT}

This study was supported by Russian Science Foundation project No. 19-74-10056.

\section{REFERENCES}

[1] Labinskyy N., Csiszar A., Orosz Z., et al. Comparison of endothelial function, $\mathrm{O} 2 \cdot$ and $\mathrm{H} 2 \mathrm{O} 2$ production, and vascular oxidative stress resistance between the longest-living rodent, the naked mole rat, and mice. Am. J. Physiol. Heart. Circ. Physiol. 2006; 291: 2698-2704.

[2] Wyatt M.D., Pittman D.L. Methylating agents and DNA repair responses: Methylated bases and sources of strand breaks. Chemical Research in Toxicology. 2006;19(12):1580-94.

[3] Longley D.B., Harkin D.P., Johnston P.G. 5-fluorouracil: mechanisms of action and clinical strategies. Nat. Rev. Cancer. 2003; 3:330-338.

[4] Hande K.R. Etoposide: four decades of development of a topoisomerase II inhibitor. Eur. J. Cancer. 1998; 34 (10): 1514-21. 